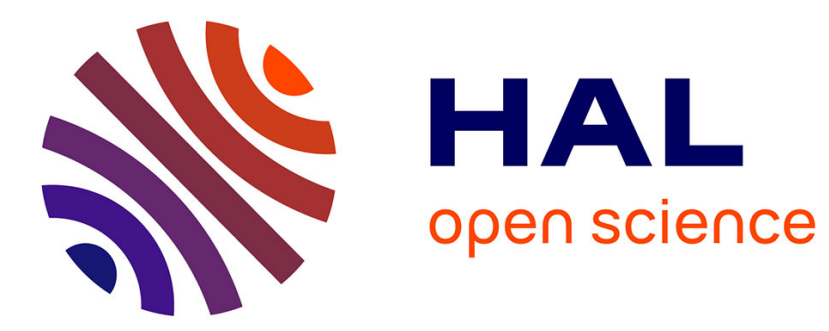

\title{
Pouvoir réflecteur d'une lame diélectrique en propagation guidée application à la mesure des permittivités complexes
}

\author{
J.L. Miane
}

\section{> To cite this version:}

J.L. Miane. Pouvoir réflecteur d'une lame diélectrique en propagation guidée application à la mesure des permittivités complexes. Revue de Physique Appliquée, 1969, 4 (1), pp.45-49. 10.1051/rphysap:019690040104500 . jpa-00242889

\section{HAL Id: jpa-00242889 https://hal.science/jpa-00242889}

Submitted on 1 Jan 1969

HAL is a multi-disciplinary open access archive for the deposit and dissemination of scientific research documents, whether they are published or not. The documents may come from teaching and research institutions in France or abroad, or from public or private research centers.
L'archive ouverte pluridisciplinaire $\mathbf{H A L}$, est destinée au dépôt et à la diffusion de documents scientifiques de niveau recherche, publiés ou non, émanant des établissements d'enseignement et de recherche français ou étrangers, des laboratoires publics ou privés. 


\title{
POUVOIR RÉFLEGTEUR D’UNE LAME DIÉLEGTRIQUE EN PROPAGATION GUIDÉE APPLIGATION A LA MESURE DES PERMITTIVITÉS COMPLEXES
}

\author{
Par J. L. MIANE, \\ Laboratoire de Physique Expérimentale, Faculté des Sciences de Bordeaux.
}

(Reçu le 20 mars 1968.)

\begin{abstract}
Résumé. - La détermination de la permittivité complexe est obtenue à partir de la mesure du pouvoir réflecteur d'une lame diélectrique (ou semiconductrice) placée en avant d'un courtcircuit mobile.

Le pouvoir réflecteur $R$ est une fonction périodique de la distance $\Delta$ séparant le diélectrique du court-circuit et ses extremums $R_{\mathrm{M}}$ et $R_{\mathrm{m}}$ ne dépendent que de la permittivité du diélectrique. La mesure de $R_{\mathrm{M}}$ et $R_{\mathrm{m}}$ conduit à la détermination de $\varepsilon^{\prime}$ et $\varepsilon^{\prime \prime}$.

Abstract. - In the technique describe in this paper, measurement of complex permittivity is accomplished by measuring the power reflection factor of a solid dielectric (or semiconductor) sheet in a waveguide terminated by a micrometer plunger.

The power reflection factor $R$ is oscillating in $\Delta$ ( $\Delta$ is the distance between the sheet and the movable short-circuit) and its extrema $R_{\mathbf{M}}$ and $R_{\mathrm{m}}$ are functions only of the complex permittivity of the dielectric. Measurements of $R_{\mathrm{M}}$ and $R_{\mathrm{m}}$ provide a means of finding $\varepsilon^{\prime}$ and $\varepsilon^{\prime \prime}$ at ultra high frequencies.
\end{abstract}

I. Introduction. - Les méthodes de mesure valables dans la bande $\mathrm{X}$ (méthode du court-circuit, cavités résonnantes, etc. [1]) ne peuvent pas être transposées sans de grandes difficultés aux bandes de fréquences millimétriques. Il est donc nécessaire d'élaborer d'autres méthodes.

A ces fréquences, les lignes de mesures ne permettent plus une mesure précise des impédances ni des coefficients de réflexion complexes : seul le carré module du coefficient de réflexion (pouvoir réflecteur) peut être mesuré avec précision par des mesures de puissances ou d'atténuations.

Nous allons montrer que, sous certaines conditions

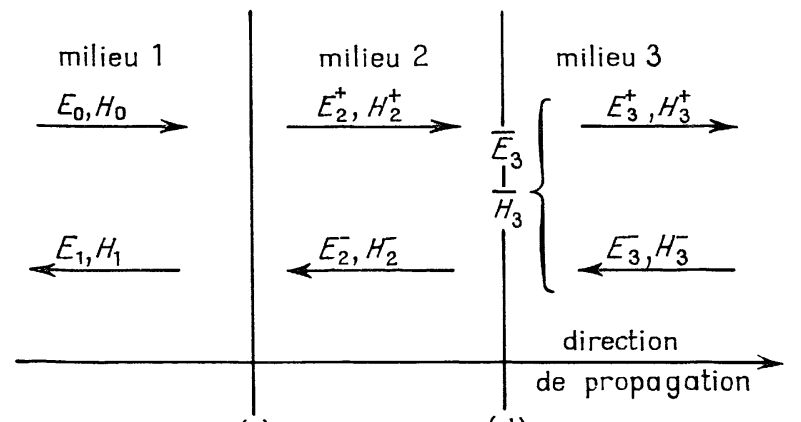

(o)

(d)

face d'entrée face de sortie

FIG. 1. - Réflexion et transmission d'une onde plane par une lame diélectrique sous incidence normale. qui seront précisées, la mesure du pouvoir réflecteur d'une lame diélectrique conduit directement à la détermination de la permittivité complexe.

II. Réflexion sur une lame diélectrique. - a) REPORT DES COEFFICIENTS DE RÉFLEXION. - Nous envisagerons d'abord le cas d'une propagation libre, les résultats obtenus pourront ensuite être étendus au cas de la propagation guidée (mode $\mathrm{TE}_{01}$ ).

La lame diélectrique 2 est placée entre les milieux 1 et 3 (fig. 1). Nous supposerons que dans le milieu 3 existe une onde réfléchie $\left(E_{3}^{-}, H_{3}^{-}\right)$.

Les conditions aux limites sur les faces $(0)$ et (d) de la lame conduisent aux relations suivantes :

(d) $\left\{\begin{array}{c}E_{2}^{+} \exp \left(-\gamma_{2} d\right)+E_{2}^{-} \exp \left(\gamma_{2} d\right) \\ \quad=E_{3}^{+} \exp \left(-\gamma_{3} d\right)+E_{3}^{-} \exp \left(\gamma_{3} d\right)=\bar{E}_{3} \\ \frac{1}{Z_{2}}\left[E_{2}^{+} \exp \left(-\gamma_{2} d\right)-E_{2}^{-} \exp \left(\gamma_{2} d\right)\right] \\ \quad=H_{3}^{+} \exp \left(-\gamma_{3} d\right)+H_{3}^{-} \exp \left(\gamma_{3} d\right)=\bar{H}_{3}\end{array}\right.$

$$
\text { (0) }\left\{\begin{array}{l}
E_{0}+E_{1}=E_{2}^{+}+E_{2}^{-} \\
E_{0}-E_{1}=\frac{Z_{1}}{Z_{2}}\left(E_{2}^{+}-E_{2}^{-}\right)
\end{array}\right.
$$

où $\bar{E}_{3}$ et $\bar{H}_{3}$ représentent les amplitudes complexes sur la surface $(d), Z_{1}$ et $Z_{2}$ les impédances caractéristiques des milieux 1 et 2 , $\gamma_{2}$ et $\gamma_{3}$ les constantes de propagation dans les milieux 2 et 3 . 
Le rapport $\bar{E}_{3} / \bar{H}_{3}$ définit l'impédance d'onde $\bar{Z}_{3}$ sur la surface $(d)$. La solution de ce système donne le coefficient de réflexion $E_{1} / E_{0}$. En posant $r_{12}=\frac{Z_{2}-Z_{1}}{Z_{2}+Z_{1}}$ et $r_{23}=\frac{\bar{Z}_{3}-Z_{2}}{\bar{Z}_{3}+Z_{2}}$, nous obtiendrons :

$$
\frac{E_{1}}{E_{0}}=\rho=\frac{r_{12}+r_{23} \exp \left(-2 \gamma_{2} d\right)}{1+r_{12} r_{23} \exp \left(-2 \gamma_{2} d\right)}
$$

si le trièdre $\left(\bar{E}_{3}, \bar{H}_{3}, \mathbf{k}\right)$ est direct, où $\mathbf{k}$ représente le vecteur unitaire de la direction de propagation.

Dans le cas où le trièdre $\left(\bar{E}_{3}, \bar{H}_{3}, \mathbf{k}\right)$ est inverse :

$$
\frac{E_{1}}{E_{0}}=\rho=\frac{1+r_{12} r_{23} \exp \left(-2 \gamma_{2} d\right)}{r_{12}+r_{23} \exp \left(-2 \gamma_{2} d\right)} .
$$

b) Pouvoir Réflegteur. - Nous définirons le pouvoir réflecteur $R$ comme le rapport de l'énergie réfléchie à l'énergie incidente :

$$
R=\left(\frac{E_{1}}{E_{0}}\right)^{2}=|\rho|^{2} .
$$

Posons $r_{12}=\mathrm{e}^{-2(s+j \varphi)}$ et $r_{23}=\mathrm{e}^{-2(n+j \xi)}$; en portant ces expressions dans la formule (1) ou $\left(1^{\prime}\right)$, nous obtenons une nouvelle forme pour $\rho$ :

$\rho=\frac{\exp [-2(s+j \varphi)]+\exp \left[-2\left(\eta+j \xi+\gamma_{2} d\right)\right]}{1+\exp \left[-2\left(s+\eta+j \xi+j \varphi+\gamma_{2} d\right)\right]}$

soit en multipliant haut et bas par

$$
\exp \left(s+\eta+j \xi+j \varphi+\gamma_{2} d\right)
$$

et en explicitant $\gamma_{2}$ qui, en général, sera complexe :

$$
\begin{gathered}
\gamma_{2}=\alpha_{2}+j \beta_{2} \\
\rho=\frac{\operatorname{ch}\left[\left(\eta-s+\alpha_{2} d\right)+j\left(\xi-\varphi+\beta_{2} d\right)\right]}{\operatorname{ch}\left[\left(\eta+s+\alpha_{2} d\right)+j\left(\xi+\varphi+\beta_{2} d\right)\right]} .
\end{gathered}
$$

Le pouvoir réflecteur $R$ s'exprime alors en fonction des six paramètres $\alpha_{2}, \beta_{2}, \eta, s, \xi$ et $\varphi$ :

$R=\frac{\operatorname{sh}^{2}\left(\eta-s+\alpha_{2} d\right)+\cos ^{2}\left(\xi-\varphi+\beta_{2} d\right)}{\operatorname{sh}^{2}\left(\eta+s+\alpha_{2} d\right)+\cos ^{2}\left(\xi+\varphi+\beta_{2} d\right)}$.

Ces six paramètres ne sont pas indépendants : ils s'expriment, à leur tour, en fonction des propriétés des milieux 1,2 et 3 . Si, en particulier, les propriétés des milieux 1 et 3 sont connues, ces paramètres ne dépendent plus que de la permittivité complexe $\left(\varepsilon^{\prime}-j \varepsilon^{\prime \prime}\right)$ du diélectrique 2 ; on peut donc penser qu'un minimum de deux mesures du pouvoir réflecteur dans des conditions expérimentales différentes permettra de déterminer $\varepsilon^{\prime}$ et $\varepsilon^{\prime \prime}$. Si nous revenons à l'expression de $R$, les termes susceptibles d'être modifiés sont : l'épaisseur $d$ et le milieu 3 , soit $\eta$ et $\xi$ (il suffit de modifier la terminaison de la ligne en propagation guidée).

- Pour une terminaison donnée, il est possible de faire varier $d$ : ceci dans le cas de liquides. Sur ce principe sont basées les méthodes décrites par Boyer [2] et Garg, Kilp, Smyth [3].

- Dans le cas d'un solide, ces méthodes ne sont plus applicables : il est alors possible d'agir sur la terminaison de la ligne [4].

C'est sur cette idée que repose la méthode de mesure que nous allons décrire : la terminaison de la ligne sera constituée d'une longueur $\Delta$ de guide et d'un court-circuit.

III. Pouvoir réflecteur d'une lame diélectrique placée en avant d'un court-circuit. - En propagation guidée, les équations précédemment obtenues restent valables : $Z_{1}$ et $Z_{2}$ représentent alors les impédances caractéristiques guidées dans les milieux 1,2 , et $\bar{Z}_{3}$ l'impédance d'onde guidée sur la surface $(d)$ de la lame diélectrique.

a) EXPRESSION DES PARAMÈTRES $s$ ET $\varphi$. - Nous exprimerons d'abord $r_{12}$ en fonction des propriétés des milieux 1 et 2 .

En remarquant que, pour des milieux non magnétiques $\left(\mu=\mu_{0}\right) Z_{n} \gamma_{n}=$ Cte, nous pouvons écrire :

$$
r_{12}=\frac{Z_{2}-Z_{1}}{Z_{2}+Z_{1}}=\frac{\gamma_{1}-\gamma_{2}}{\gamma_{1}+\gamma_{2}} \text {. }
$$

En propagation guidée, nous avons :

$$
\gamma^{2}=-\frac{4 \pi^{2}}{\lambda_{0}^{2}}\left[\varepsilon_{\mathrm{r}}-\left(\frac{\lambda_{0}}{\lambda_{\mathrm{c}}}\right)^{2}\right]=(\alpha+j \beta)^{2}
$$

où $\lambda_{0}$ représente la longueur d'onde dans le vide, $\lambda_{\mathrm{c}}$ la longueur d'onde de coupure du guide utilisé et $\varepsilon_{\mathrm{r}}$ la permittivité relative du milieu considéré.

Pour le milieu 1, le guide est rempli d'air : $\varepsilon_{\mathrm{r}}=1$.

Pour le milieu $2: \varepsilon_{\mathrm{r}}=\varepsilon^{\prime}-j \varepsilon^{\prime \prime}$.

Nous obtenons donc pour $\alpha$ et $\beta$, en développant au $1^{\text {er }}$ ordre $\left(\varepsilon^{\prime \prime} / \varepsilon^{\prime}\right.$ est petit devant 1$)$ :

$$
\begin{aligned}
& \alpha=\frac{\pi}{\lambda_{0}} \frac{\varepsilon^{\prime \prime}}{\left[\varepsilon^{\prime}-\left(\frac{\lambda_{0}}{\lambda_{\mathrm{c}}}\right)^{2}\right]^{1 / 2}}\left\{1-\frac{1}{8}\left[\frac{\varepsilon^{\prime \prime}}{\varepsilon^{\prime}-\left(\frac{\lambda_{0}}{\lambda_{\mathrm{c}}}\right)^{2}}\right]^{2}\right\} \\
& \beta=\frac{2 \pi}{\lambda_{0}}\left[\varepsilon^{\prime}-\left(\frac{\lambda_{0}}{\lambda_{\mathrm{c}}}\right)^{2}\right]^{1 / 2}\left\{1+\frac{1}{8}\left[\frac{\varepsilon^{\prime \prime}}{\varepsilon^{\prime}-\left(\frac{\lambda_{0}}{\lambda_{\mathrm{c}}}\right)^{2}}\right]^{2}\right\}
\end{aligned}
$$

Nous limiterons cette étude à des milieux à pertes

suffisamment faibles pour que
négligeable devant 1.
Nous poserons :

$$
\begin{array}{ll}
\gamma_{2}=\alpha_{2}+j \beta_{2} & \text { (milieu à pertes) } \\
\gamma_{1}=j \beta_{\mathrm{g}} & \text { (milieu sans pertes). }
\end{array}
$$

Alors :

$$
r_{12}=\frac{-\alpha_{2}+j\left(\beta_{\mathrm{g}}-\beta_{2}\right)}{\alpha_{2}+j\left(\beta_{\mathrm{g}}+\beta_{2}\right)} .
$$


Nous pourrons déterminer $s$ à partir du module de $r_{12}$ :

$$
r_{12}^{2}=\frac{\alpha_{2}^{2}+\left(\beta_{\mathrm{g}}-\beta_{2}\right)^{2}}{\alpha_{2}^{2}+\left(\beta_{\mathrm{g}}+\beta_{2}\right)^{2}} .
$$

La comparaison des différents termes montre que $\alpha_{2}^{2}$ est négligeable devant $\left(\beta_{\mathrm{g}} \pm \beta_{2}\right)^{2}$.

Donc :

$$
\left|r_{12}\right|=\frac{\beta_{2}-\beta_{\mathrm{g}}}{\beta_{2}+\beta_{\mathrm{g}}} .
$$

En remplaçant $\beta_{2}$ et $\beta_{\mathrm{g}}$ par leur valeur, et en posant : $K^{\prime}=\frac{\varepsilon^{\prime}-\left(\lambda_{0} / \lambda_{c}\right)^{2}}{1-\left(\lambda_{0} / \lambda_{c}\right)^{2}}$, nous obtenons finalement l'expression de $s$ :

$$
\mathrm{e}^{-2 s}=\left|r_{12}\right|=\frac{\sqrt{K^{\prime}}-1}{\sqrt{K^{\prime}}+1} .
$$

D'une manière analogue, nous déterminerons à partir de $\operatorname{Arg}\left(r_{12}\right)$ :

$-2 \varphi=\operatorname{Arg}\left(r_{12}\right)=\operatorname{Arctg} \frac{\beta_{2}-\beta_{\mathrm{g}}}{\alpha_{2}}-\operatorname{Arctg} \frac{\beta_{2}+\beta_{\mathrm{g}}}{\alpha_{2}}$.

Dans les mêmes conditions que précédemment ( $\alpha_{2}^{2}$ négligeable devant $\left.\left(\beta_{2} \pm \beta_{\mathrm{g}}\right)^{2}\right)$, nous obtenons :

$$
\varphi=-\frac{1}{2} \operatorname{Arg}\left(r_{12}\right)=\frac{\alpha_{2} \beta_{\mathrm{g}}}{\beta_{2}^{2}-\beta_{\mathrm{g}}^{2}} .
$$

Dans le cas limite où $\varepsilon^{\prime \prime}=0$ (réflexion sur un milieu sans pertes), nous savons que $\operatorname{Arg}\left(r_{12}\right)=\pi$, ce qui conduit à choisir la détermination suivante pour $\varphi$ :

$$
\begin{aligned}
\varphi=\frac{\pi}{2}+ & \frac{\alpha_{2} \beta_{\mathrm{g}}}{\beta_{2}^{2}-\beta_{\mathrm{g}}^{2}} \\
& =\frac{\pi}{2}+\frac{1}{2} \frac{\varepsilon^{\prime \prime}}{1-\left(\lambda_{0} / \lambda_{\mathrm{c}}\right)^{2}} \cdot \frac{1}{\sqrt{K^{\prime}}\left(K^{\prime}-1\right)} .
\end{aligned}
$$

Le terme $\frac{\varepsilon^{\prime \prime}}{\sqrt{K^{\prime}}\left(K^{\prime}-1\right)}$ pourra généralement être négligé : donc en première approximation $\varphi=\pi / 2$.

b) EXPRESSION DES PARAMÈTRES $\eta$ ET $\xi$. - Le coefficient de réflexion $r_{23}$ peut se calculer à partir de la formule (1). La figure 2 représente la terminaison que nous allons étudier : un court-circuit est placé à la distance $\Delta$ de la face « de sortie » de la lame

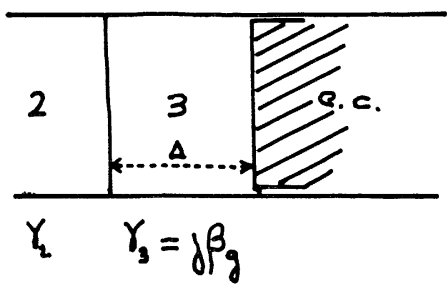

FIG. 2. - Terminaison réfléchissante : c.c., court-circuit 2 , lame diélectrique ou semiconductrice. diélectrique. Le coefficient de réflexion sur le dioptre 2-3 est $-r_{12}=-\exp [-2(s+j \varphi)]$ et sur le courtcircuit : -1 .

Donc le coefficient $r_{23}$, compte tenu des réflexions multiples, sera (dans le milieu $3: \gamma_{3}=j \beta_{\mathrm{g}}$ ):

$$
r_{23}=-\frac{r_{12}+\exp \left(-2 j \beta_{\mathrm{g}} \Delta\right)}{1+r_{12} \exp \left(-2 j \beta_{\mathrm{g}} \Delta\right)}
$$

en exprimant $r_{12}$ en fonction de $s$ et $\varphi$, on obtient : $\begin{array}{lll}r_{23}=-\frac{\operatorname{ch}\left[s+j\left(\varphi+\beta_{\mathrm{g}} \Delta\right)\right]}{\operatorname{ch}\left[s+j\left(\varphi-\beta_{\mathrm{g}} \Delta\right)\right]} & \text { si } & 0<\beta_{\mathrm{g}} \Delta<\frac{\pi}{2} \\ r_{23}=-\frac{\operatorname{ch}\left[s+j\left(\varphi-\beta_{\mathrm{g}} \Delta\right)\right]}{\operatorname{ch}\left[s+j\left(\varphi+\beta_{\mathrm{g}} \Delta\right)\right]} & \text { si } & \frac{\pi}{2}<\beta_{\mathrm{g}} \Delta<\pi .\end{array}$

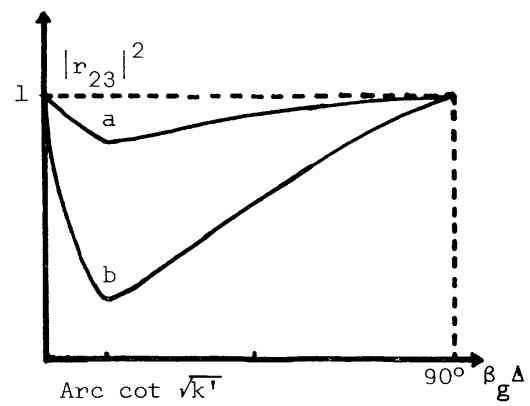

FIG. 3. - Variations de $\left|r_{23}\right|^{2}$ en fonction de $\Delta\left(\varepsilon^{\prime}=16\right)$ :

$$
\frac{\varepsilon^{\prime \prime}}{\varepsilon^{\prime}-\left(\lambda_{0} / \lambda_{\mathrm{c}}\right)^{2}}=0,16(\mathrm{a}), 1,5(\mathrm{~b}) \text {. }
$$

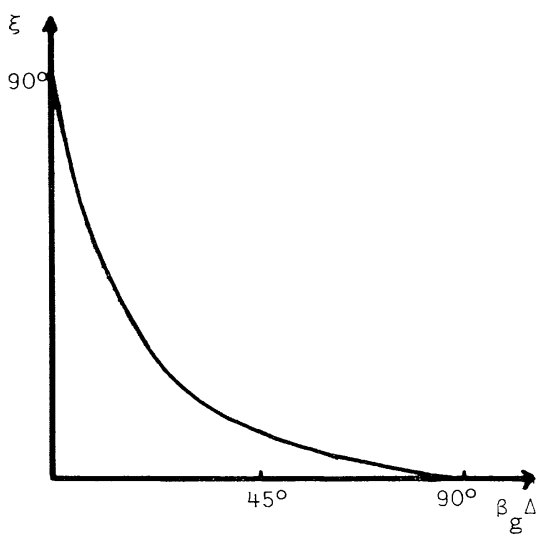

FIG. 4. - Variation de $\xi$ en fonction de $\Delta\left(\varepsilon^{\prime}=16\right)$.

Les figures 3 et 4 représentent les variations de $\left|r_{23}\right|^{2}$ et de $\xi=-\frac{1}{2} \operatorname{Arg}\left(r_{23}\right)$ pour $0<\beta_{\mathrm{g}} \Delta<\frac{\pi}{2}$ et $\varepsilon^{\prime}=16$

$$
\left|r_{23}\right|^{2}=\frac{\operatorname{sh}^{2}(s)+\cos ^{2}\left(\varphi+\beta_{\mathrm{g}} \Delta\right)}{\operatorname{sh}^{2}(s)+\cos ^{2}\left(\varphi-\beta_{\mathrm{g}} \Delta\right)}
$$

$-2 \xi=\operatorname{Arg}\left(r_{23}\right)=\pi+\operatorname{Arctg}\left[\operatorname{th}(s) \operatorname{tg}\left(\varphi+\beta_{\mathrm{g}} \Delta\right)\right]$ $-\operatorname{Arctg}\left[\operatorname{th}(s) \operatorname{tg}\left(\varphi-\beta_{\mathrm{g}} \Delta\right)\right]$. 
La détermination de $\xi$ est fixée par les conditions aux limites si $\beta_{\mathrm{g}} \Delta=0, \operatorname{Arg}\left(r_{23}\right)=\pi$ (lame courtcircuitée). Tant que $\varepsilon^{\prime \prime} \mid \varepsilon^{\prime}$ est petit, $\left|r_{23}\right|^{2}$ reste voisin de 1 , sauf au voisinage de $\Delta^{\prime}$ tel que :

$$
\beta_{\mathrm{g}} \Delta^{\prime}=\operatorname{Arctg} \frac{1}{\sqrt{K^{\prime}}} .
$$

En général, on pourra considérer $\eta$ comme nul quitte à vérifier par la suite qu'il en est bien ainsi.

c) Pouvoir réflegteur. - En revenant à la formule (2), nous pourrons écrire si $\eta=0$ et $\varphi=\pi / 2$ :

$$
R=\frac{\operatorname{sh}^{2}\left(\alpha_{2} d-s\right)+\sin ^{2}\left(\xi+\beta_{2} d\right)}{\operatorname{sh}^{2}\left(\alpha_{2} d+s\right)+\sin ^{2}\left(\xi+\beta_{2} d\right)} .
$$

L'étude des variations de $R$ en fonction de $\left(\xi+\beta_{2} d\right)$ montre que $R$ passe :

par un maximum :

$R_{\mathrm{M}}=\frac{\mathrm{ch}^{2}\left(\alpha_{2} d-s\right)}{\mathrm{ch}^{2}\left(\alpha_{2} d+s\right)}$ pour $\xi+\beta_{2} d=\frac{\pi}{2}(+k \pi)$

par un minimum :

$R_{\mathrm{m}}=\frac{\mathrm{sh}^{2}\left(\alpha_{2} d-s\right)}{\mathrm{sh}^{2}\left(\alpha_{2} d+s\right)}$ pour $\xi+\beta_{2} d=0(+k \pi)$

$\alpha_{2}$ et $s s^{\prime}$ expriment en fonction de $\varepsilon^{\prime}$ et $\varepsilon^{\prime \prime}$. De la mesure des pouvoirs réflecteurs $R_{\mathrm{M}}$ et $R_{\mathrm{m}}$, il sera possible de déduire $\varepsilon^{\prime}$ et $\varepsilon^{\prime \prime}$, par l'intermédiaire de $\alpha_{2}$ et $s$.

Si $d>s / \alpha_{2}$ :

$$
\begin{aligned}
\operatorname{th}^{2}\left(\alpha_{2} d\right) & =\frac{\left(1-\sqrt{R_{\mathrm{M}}}\right)\left(1+\sqrt{R_{\mathrm{m}}}\right)}{\left(1+\sqrt{R_{\mathrm{M}}}\right)\left(1-\sqrt{\overline{R_{\mathrm{m}}}}\right)} \\
\operatorname{th}^{2}(s) & =\frac{\left(1-\sqrt{R_{\mathrm{M}}}\right)\left(1-\sqrt{R_{\mathrm{m}}}\right)}{\left(1+\sqrt{R_{\mathrm{M}}}\right)\left(1+\sqrt{R_{\mathrm{m}}}\right)} .
\end{aligned}
$$

IV. Application à la mesure des permittivités complexes. - a) SGHÉmA du montAge. - La mesure du pouvoir réflecteur se ramène à une mesure de puissance, le schéma de principe du montage utilisé pour cette mesure est représenté sur la figure 5 .

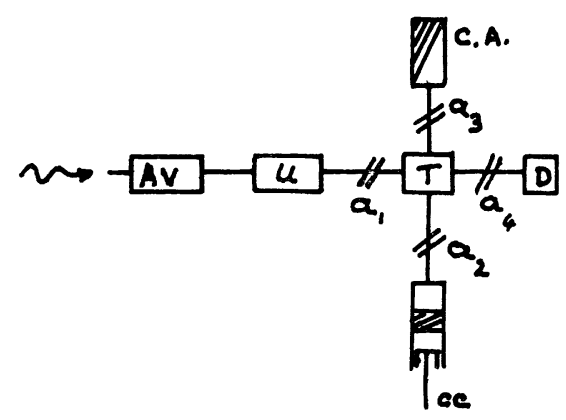

FIG. 5. - Schéma du montage : Av, atténuateur étalonné ; u, guide unidirectionnel; T, Té hybride ; C.A., charge adaptée; c.c., court-circuit variable; D, détecteur; $a_{i}$, adaptateurs.
La puissance réfléchie sur l'échantillon est séparée de l'onde incidente et dirigée vers un détecteur par un Té.

De la mesure de $R_{\mathrm{M}}$ et $R_{\mathrm{m}}$, on tire $s$ et $\alpha_{2}$ puis $\varepsilon^{\prime}$ et $\varepsilon^{\prime \prime}$ à l'aide des formules (6) et (7).

b) Conditions D'Application de GetTe mÉthode. - Les pouvoirs réflecteurs $R_{\mathrm{M}}$ et $R_{\mathrm{m}}$ sont des fonctions de l'épaisseur $d$ de la lame diélectrique.

On remarque que $R_{\mathrm{m}}$ s'annule pour une épaisseur $d_{0}=s / \alpha_{2}$, où :

$$
d_{0}=\frac{\sqrt{\varepsilon^{\prime}-\left(\frac{\lambda_{0}}{\lambda_{\mathrm{c}}}\right)^{2}}}{\varepsilon^{\prime \prime}} \frac{\lambda_{0}}{2 \pi} \log \frac{\sqrt{K^{\prime}}+1}{\sqrt{K^{\prime}}-1} .
$$

La précision obtenue sur $\varepsilon^{\prime}$ et $\varepsilon^{\prime \prime}$ est fonction de la précision des mesures mais aussi de la différence $R_{\mathrm{M}}-R_{\mathrm{m}}$ qui doit être assez grande. L'épaisseur optimum est comprise entre $2 d_{0}$ et $5 d_{0}$.

Si l'épaisseur de l'échantillon devient grande, de telle façon que l'onde soit totalement absorbée dans le diélectrique, $R_{\mathrm{M}}$ et $R_{\mathrm{m}}$ tendent vers une limite commune $R_{l}=1 / \mathrm{e}^{4 s}$, ce qui permet sur un échantillon très long d'obtenir une estimation de $\varepsilon^{\prime}$.

Nous rappelons enfin que les formules $\left(5^{\prime}\right)$ et $\left(5^{\prime \prime}\right)$ qui permettent de calculer $\varepsilon^{\prime}$ et $\varepsilon^{\prime \prime}$ ne sont valables que si $\frac{1}{8}\left|\frac{\varepsilon^{\prime \prime}}{\varepsilon^{\prime}-\left(\frac{\lambda_{0}}{\lambda_{\mathrm{c}}}\right)^{2}}\right|^{2}$ est négligeable devant 1. Si les pertes deviennent trop importantes, le paramètre $\eta$ ne peut plus être négligé.

c) VÉRIFICATION EXPÉRIMENTALE DE LA THÉORIE. A titre de vérification, nous avons tout d'abord déterminé expérimentalement la fonction $R=f(\Delta)$ pour un diélectrique à pertes : la fibrine.

La mesure de $R$ a été faite à l'aide du montage de

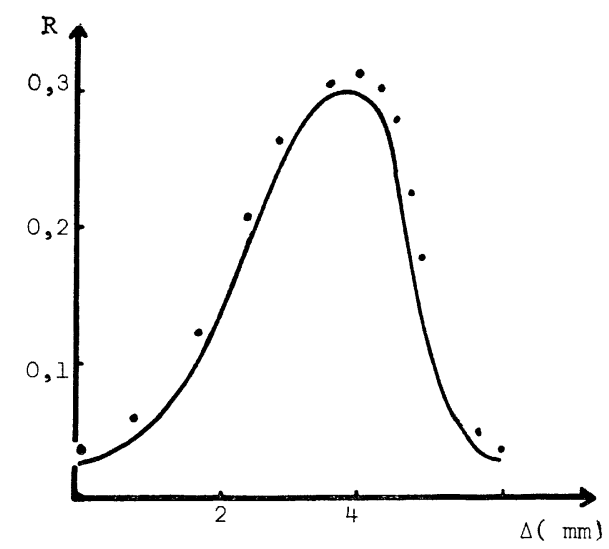

FIG. 6. - Variations théoriques et expérimentales au pouvoir réflecteur en fonction de la position du court-circuit.

En trait plein : courbe théorique $\left(\varepsilon^{\prime}=3,30, \varepsilon^{\prime \prime}=0,33\right)$. - : points expérimentaux. 
la figure 5. Le détecteur était un cristal $1 \mathrm{~N} \mathrm{23;} \mathrm{la}$ mesure des puissances se faisant par la méthode de l'atténuateur, il n'était pas nécessaire de connaître la courbe de détection du cristal.

La courbe obtenue $R=f(\Delta)$ est ensuite comparée à la courbe théorique ( $f$ ig. 6 ), où $\varepsilon^{\prime}=3,30$ et $\varepsilon^{\prime \prime}=0,33$ :

$$
R=\frac{\operatorname{sh}^{2}\left(\alpha_{2} d-s\right)+\cos ^{2}\left(\xi+\beta_{2} d-\varphi\right)}{\operatorname{sh}^{2}\left(\alpha_{2} d+s\right)+\cos ^{2}\left(\xi+\beta_{2} d+\varphi\right)}
$$

$s$ et $\xi$ sont définis par les relations (3) et (4), $\eta$ est supposé nul.

Nous avons mesuré d'autre part $\varepsilon^{\prime}$ et $\varepsilon^{\prime \prime}$ par la méthode du court-circuit et avons obtenu :

$$
\begin{aligned}
& \varepsilon^{\prime}=3,35 \pm 0,10 \\
& \varepsilon^{\prime \prime}=0,35 \pm 0,01
\end{aligned}
$$

valeurs en bon accord avec les précédentes.

Le calcul de $\eta$ avec ces valeurs montre que l'approximation faite $(\eta=0)$ était justifiée : $\eta<0,006$. Il en est de même de $\varphi$.
La courbe expérimentale obtenue coïncide avec la courbe théorique à une translation près : $R_{\mathrm{vrai}}=R_{\text {mesuré }}$ - constante, pour $\varepsilon^{\prime}=3,30$ et $\varepsilon^{\prime \prime}=0,33$, translation due aux réflexions parasites qui n'ont pu être totalement éliminées dans le Té.

V. Conclusion. - La méthode de mesure des permittivités complexes que nous venons d'exposer peut être appliquée aux semiconducteurs en remplaçant le terme $\varepsilon^{\prime \prime}$ par $\sigma / \varepsilon_{0} \omega$ où $\sigma$ représente la conductivité aux hyperfréquences du semiconducteur et $\omega$ la pulsation de l'onde.

Par ce moyen, nous avons pu déterminer la dispersion et l'absorption du germanium type $n$ dans la bande $Q[5]$ (de 30 à $36 \mathrm{GHz}$ ).

Cette méthode s'avère particulièrement commode pour des études sur des semiconducteurs ou des études en fonction de la température. En effet, il n'est pas nécessaire de manipuler l'échantillon en cours de mesure.

\section{BIBLIOGRAPHIE}

[1] VON Hippei, (A. R.), Les diélectriques et leurs applications, Dunod, Paris, 1961.

[2] Boyer (P.), J. Phys. Appl., 1965, 2 b, 201 A.

[3] Garg (S. K.), Kilp (H.) et Smyth (C. P.), J. Chem. Soc. G., 1965, B 43, 2341.
[4] Roberts (P. S.) et Mukainata (T.), Pittsburg Pa, Instrum. Soc. Amer., 1965, 42, 1.

[5] Mrane (J. L.), Thèse $3^{\text {e }}$ cycle, Spectronomie hertzienne, Bordeaux, 1967. 